\title{
Catalogue of observed tangents to the spiral arms in the Milky Way galaxy
}

\author{
Jacques P. Vallée
}

National Research Council Canada, National Science Infrastructure portfolio, Herzberg Astrophysics, 5071 West Saanich Road, Victoria, B.C., Canada V9E 2E7

Keywords: Galaxy: disk - Galaxy: structure - Galaxy: fundamental parameters ISM: kinematics and dynamics

Accepted: 2014 August 23; to appear in the Astrophysical Journal Suppl. Ser. (2014 Oct. issue)

\begin{abstract}
From the Sun's location in the Galactic disk, one can use different arm tracers (CO, HI, thermal or ionized or relativistic electrons, masers, cold and hot dust, etc) to locate a tangent to each spiral arm in the disk of the Milky Way.We present a master Catalogue of the astronomically observed tangents to the Galaxy's spiral arms, using different arm tracers from the literature. Some arm tracers can have slightly divergent results from several papers, so a mean value is taken - see Appendix for CO, HII, and masers. The Catalogue of means currently consists of 63 mean tracer entries, spread over many arms (Carina, Crux-Centaurus, Norma, Perseus origin, near 3-kpc, Scutum, Sagittarius), stemming from 107 original arm tracer entries.
\end{abstract}

Additionally, we updated and revised a previous statistical analysis of the angular offset and linear separation from the mid-arm, for each different mean arm tracer. Given enough arm tracers, and summing and averaging over all four spiral arms, one could determine if arm tracers have separate and parallel lanes in the Milky Way. This statistical analysis allows a crosscut of a galactic spiral arm to be made, confirming a recent discovery of a linear separation between arm tracers. Here, from the mid-arm's CO to the inner edge's hot dust, the arm halfwidth is about $340 \mathrm{pc}$; doubling would yield a full arm width of $680 \mathrm{pc}$. We briefly compare these observations with the predictions of many spiral arm theories, notably the density-wave theory.

\section{Introduction}

Our whole Galaxy is composed of a disk orbiting around a nuclear bulge, all happening within a dark halo. At a distance between 7.5 and $8.5 \mathrm{kpc}$ from the Galactic Center, the Sun is immersed inside the galactic disk, composed mainly of stars with the addition of dust (warm and hot), gas (molecular and atomic), ionized gas and thermal electrons, cosmic rays and relativistic electrons, and magnetic fields. For a review of some physical properties (density, temperature, etc) of the gas phases and the magnetic fields in the interstellar medium, see Heiles \& Haverkorn (2012). Near the Galactic Center, there are two coexisting bar-like structures pointed between $14^{\circ}$ to $45^{\circ}$ with respect to the line of sight of the Sun to the Galactic Center, a stellar bulge, and the usual black hole at the center (Green et al 2011 - fig.5). The majority of published papers favors a 4-arm spiral model in the galactic disk. There is still a bit of controversy left, as onesixth of all recent publications favor a 2-arm spiral structure (see Vallée 2014b). 
Here our interest lies in the disk. Massive stars, and a large number of less massive star, molecular gas and dust are preferentially located in spiral arms. In the Milky Way, a spiral arm has a mean pitch angle near $12^{\circ}$, although estimates vary slightly. Previous sketches of the Milky Way galaxy showed stellar arms arranged in a spiral form, without much details across the arms. Recently, more details have become available.

The Sun is located in the interarm region (Figure 1), between the Perseus arm and the Sagittarius arm. There is a local "armlet" (not shown here) of length about $2.2 \mathrm{kpc}$ at a galactic longitude near $77^{\circ}$ (e.g., section 9 in Vallée 2011). There is no 'molecular ring' near the Galactic Center, but a molecular region encompassing the origins of the four spiral arms (Section 3.2 in Vallée 2008; Dobbs \& Burkert 2012). The "Perseus origin arm" near the Galactic Center (Figure 1) is at times referred to as the "far 3-kpc arm" (e.g., Dame \& Thaddeus 2008 and 2011, their $-23^{\circ}$ arm at $337^{\circ}$; table 3 in García et al 2014). The "Crux" arms is often called the "Centaurus" arm. The "Cygnus" arm is often called the "outer" arm. The "Cygnus + I' arm is the continuation of the Scutum arm (Dame \& Thaddeus - Fig.4). In Figure 1 a single bar is sketched (but no bulge), since the position angle of both bars are still loosely defined or explained (Martinez-Valpuesta 2013, theirFig.1; Romero-Gómez et al 2013, their fig.1). We know from the measured line-of-sight velocities at longitude $1=0^{\circ}$ that the near 3 -kpc arm is expanding at $-50 \mathrm{~km} / \mathrm{s}$ (close to the origin of the Norma arm), and that the far 3-kpc arm is expanding at $+50 \mathrm{~km} / \mathrm{s}$ (close to the start of the Sagittarius arm). The author is not aware of any similar velocity measurement close to the start of the 'Perseus origin' arm, nor of the start of the Scutum arm.

There have been numerous published results for the spiral arms of the Milky Way. With the Sun in the Milky Way disk, the majority of published observational results have focused on small, different parts of our Galaxy, using different arm tracers, listing the arm shape (logarithmic or wavy or polynomial), the arm pitch angle (from the circular tangent), the number of arms ( 2 or 4 or 6 ), and the interarm separation through the Sun (around $3 \mathrm{kpc}$ ). Given conflicting results over time, statistics on arm parameters were done for the period 1980-onward, catalogued in blocks of 15 to 20 each (for the blocks, see Vallée 1995, 2002, 2005, 2008, 2013, 2014a, 2014b). A composite map of all these small-scale views could approximate the mid-scale view - see Figure 1. 


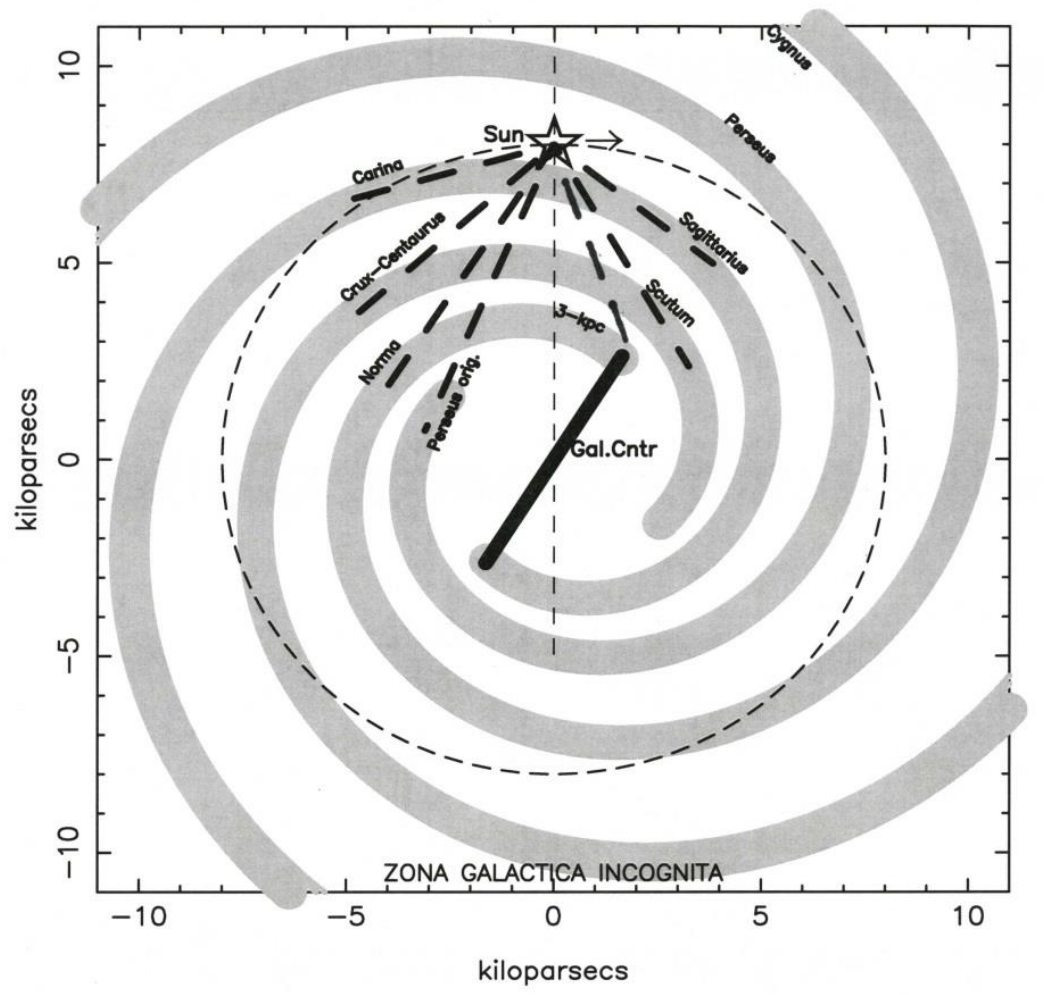

Figure 1. Old galactography. The sun is shown (open star) at $8 \mathrm{kpc}$ from the Galactic Center, with the clockwise galactic rotation (arrow). Most stars in the Milky Way galaxy are located along 4 spiral arms (gray). Other component such as clouds, gas, atoms, dust, cosmic rays are also in there. Approximate arm tangents as seen from the sun are shown (thick black dashes). The sun's orbit is shown here as a circle (thin dashes). A rough position for the Galactic bar is shown. Not much is known below the Galactic Center, termed 'Zona Galactica Incognita' a.k.a. 'unknown galactic area' following Vallée (2002, his figure 2). The Galactic Center is at $(0,0)$ and the distance scale at bottom and to the left follows common conventions.

\section{Catalogue of Galactic arm tangents in the Milky Way since 1980}

Differing ratios of components (star, gas, dust, cosmic-ray, magnetic field) can be found along an arm; some arm "segments" may have more stars while others further on may have more molecular gas. Thus the fixed view from the Sun, along the galactic disk, can miss an arm segment if using a single filter (only CO data in one segment, or only stellar counts in another segment). If we could 'move' the Sun at different positions (segments) along the same arm, we may see different local views (differing component ratios) in optical (star), near infrared (dust), or radio wavelengths (relativistic or thermal electrons, molecular gas). It is thus necessary to use many tracers when talking about a single arm. 
Small tables of arm tangents have been published before. Table 1 in Englmaier \& Gerhard (1999) had 32 entries of tracer tangents, but none for the Carina arm near $284^{\circ}$, their 'inner Galaxy' column mixed together the Scutum arm and the inner 3-kpc arm , and their far '3-kpc' arm near $-21^{\circ}$ is now called the 'Perseus origin' arm. Table 2 in Vallée (2008) had 39 tracer entries, Table 1 in Vallée (2012) had 12 tracer entries, while Table 3 in Vallée (2014a) had 43 tracer entries.

Here we embarked on an extensive literature review, to glean more arm tangents from different tracers. We recorded the complete published reference of each tracer (journal, table, figure, section), and thus were able to delete or replace erroneous or misplaced references. Also, newer instrumentation allows the detection of the arm tangents as inferred from the recent 6.7 $\mathrm{GHz}$ methanol maser or water maser data. The master catalogue of published galactic longitudes of arm tangents is given in Table 1. In Table 1, an arm is defined and labeled in only one Galactic Quadrant (I or IV); its continuation in another quadrant has another name, and we listed 63 different entries of the means of different tracer tangents (one specific tracer entry per arm), about half more than in Vallée (2014a). As much as 17 of these 63 different entries are themselves a mean of two or more published values. In addition, in Appendix A, we provided 38 individual CO entries (Table 3), 13 individual HII entries (Table 4), and 10 individual maser entries (Table 5). Thus, all in all, we catalogued 107 different tracer entries over these four tables $(63-17+38+13+10)$. For the Centaurux-Crux arm, the $\mathrm{CO}$ arm tangent was originally given as $310^{\circ}$ by Bronfman et al (1988 - fig. 7), then appeared as a typo as $300^{\circ}$ in Bronfman (1992 - his Fig.6), and was later given as $309^{\circ}$ in Garcia et al (2014 - their Fig. 14); in Appendix A we entered only the original value of $310^{\circ}$ from the first publication in 1988 .

We provide here a short $\mathrm{CO}$ analysis of the published results. The Columbia CO Surveys made at low angular resolution $(\sim 8$ arcmin) of the low excitation $(\mathrm{J}=1-0)$, low temperature $(\sim 10$ $\mathrm{K}),{ }^{12} \mathrm{CO}$ tracer (emitting at $115 \mathrm{GHz}$ ), as integrated over a velocity range associated with a spiral arm, is useful to indicate the middle of the arm. For the Carina arm, the CO tracer is given in the literature with a longitude varying from $280^{\circ}$ to $283^{\circ}$, depending on the different mathematical analyses done. The different $\mathrm{CO}$ analyses gave slightly different output values from roughly the same observed data, especially when using different averaging bin sizes in galactic longitudes (asymmetric amplitude with galactic longitude), or using different input data cutoff (63\% removal, representing the disk emission with longitude - see Fig. 14 in García et al 2014), or using a simple or complex disk emission model (Section 2 in Bronfman 1992). Of all the CO tracers used as arm tangents, the longitude-velocity analysis of García et al (2014) gave the largest longitude values for the Crux-Centaurus arm, Norma arm, and Perseus Origin (3-kpc) arm (their Table 3). They noted that their arms were first defined and delineated in the longitudevelocity diagram (their Fig. 9), and later transposed and averaged in the longitude-distance diagram. Such an analysis is bound to use somewhat arbitrary boundaries in the longitudevelocity diagram (removing some reassigned arm clouds), and the arm tangent obtained later in the other diagram with the transposed clouds could have a bigger error bar. The low resolution (8') Columbia CO survey (linked to the mid-arm) does differ as it should from the high resolution (50") Stoney Brook survey of warm CO cores (linked to new star forming regions Solomon et al 1985). 
Table 1 - Catalogue of published different spiral arm tracers (since 1980), with only one mean tracer value for each arm

\begin{tabular}{|c|c|c|c|c|c|}
\hline $\begin{array}{l}\text { Arm } \\
\text { Name }\end{array}$ & $\begin{array}{l}\text { Chemical } \\
\text { tracer }\end{array}$ & $\begin{array}{l}\text { Gal. } \\
\text { longit. } \\
\text { of arm } \\
\text { tangent(1) }\end{array}$ & $\begin{array}{l}\text { Ang. } \\
\text { dist.(2) } \\
\text { to }{ }^{12} \mathrm{CO}\end{array}$ & $\begin{array}{l}\text { Linear } \\
\text { separation } \\
\text { inside } \operatorname{arm}^{(3)} \\
\text { from }{ }^{12} \mathrm{CO}\end{array}$ & References(4) \\
\hline Carina & $\begin{array}{l}{ }^{12} \mathrm{CO} \text { at } 8 \\
\text { Thermal electron } \\
\text { HII complex } \\
\text { Dust } 240 \mu \mathrm{m} \\
\text { Dust } 60 \mu \mathrm{m} \\
\text { FIR [CII] \& [NII] }\end{array}$ & $\begin{array}{l}282^{\circ} \\
283^{\circ} \\
284^{\circ} \\
284^{\circ} \\
285^{\circ} \\
287^{\circ}\end{array}$ & $\begin{array}{l}0^{\circ} \\
10 \\
20 \\
2^{0} \\
3^{\circ} \\
5^{\circ}\end{array}$ & $\begin{array}{l}0 \mathrm{pc} \text {, at } 5 \mathrm{kpc}^{(3)} \\
87 \mathrm{pc} \\
174 \mathrm{pc} \\
174 \mathrm{pc} \\
262 \mathrm{pc} \\
435 \mathrm{pc}\end{array}$ & $\begin{array}{l}\text { Bronfman et al (2000b - table 2); see Table } 3 \\
\text { Taylor \& Cordes ( } 1993 \text { - fig. } 4) \\
\text { Russeil (2003 - table } 6) ; \text { see Table } 4 \\
\text { Drimmel ( } 2000 \text {-fig. } 1) \\
\text { Bloemen et al ( } 1990 \text { - fig.5) } \\
\text { Steiman-Cameron et al (2010 - sect. } 2.1)\end{array}$ \\
\hline $\begin{array}{l}\text { Crux- } \\
\text { Cen- } \\
\text { taurus }\end{array}$ & $\begin{array}{l}{ }^{12} \mathrm{CO} \text { at } 8 \\
\text { Thermal electron } \\
\text { HII complex } \\
\text { FIR [CII] \& [NII] } \\
\text { HI atom } \\
{ }^{26} \mathrm{Al} \\
\text { Sync. } 408 \mathrm{MHz} \\
\text { Dust } 240 \mu \mathrm{m} \\
\text { Dust } 60 \mu \mathrm{m} \\
\text { Dust } 870 \mu \mathrm{m}\end{array}$ & $\begin{array}{l}309^{\circ} \\
309^{\circ} \\
309^{\circ} \\
309^{\circ} \\
310^{\circ} \\
310^{\circ} \\
310^{\circ} \\
311^{\circ} \\
311^{\circ} \\
311^{\circ}\end{array}$ & $\begin{array}{l}00 \\
00 \\
00 \\
00 \\
10 \\
10 \\
10 \\
20 \\
20 \\
20\end{array}$ & $\begin{array}{l}0 \mathrm{pc} \text {, at } 6 \mathrm{kpc}^{(3)} \\
0 \mathrm{pc} \\
0 \mathrm{pc} \\
0 \mathrm{pc} \\
105 \mathrm{pc} \\
105 \mathrm{pc} \\
105 \mathrm{pc} \\
209 \mathrm{pc} \\
209 \mathrm{pc} \\
209 \mathrm{pc}\end{array}$ & $\begin{array}{l}\text { Bronfman et al (2000b - table 2); see Table } 3 \\
\text { Taylor \& Cordes (1993 - fig.4) } \\
\text { Russeil (2003 - table 6); see Table } 4 \\
\text { Steiman-Cameron et al (2010 - sect. 2.1) } \\
\text { Englmaier \& Gerhard (1999- table 1) } \\
\text { Chen et al (1996- fig.1) } \\
\text { Beuermann et al (1985 - fig.1) } \\
\text { Drimmell (2000 - fig. 1) } \\
\text { Bloemen et al (1990 - fig.5) } \\
\left.\text { Beuther et al (2012 - fig.2; another peak at } 305^{\circ}\right)\end{array}$ \\
\hline Norma & 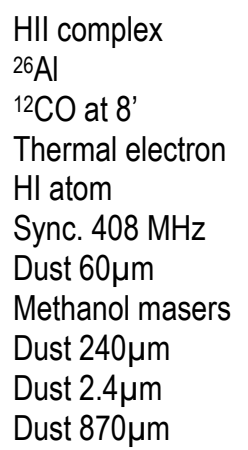 & $\begin{array}{l}325^{\circ} \\
325^{\circ} \\
328^{\circ} \\
328^{\circ} \\
328^{\circ} \\
328^{\circ} \\
329^{\circ} \\
331.5^{\circ} \\
332^{\circ} \\
332^{\circ} \\
332^{\circ}\end{array}$ & $\begin{array}{l}-30 \\
-30 \\
00 \\
00 \\
00 \\
00 \\
10 \\
3.5^{\circ} \\
40 \\
40 \\
40\end{array}$ & $\begin{array}{l}-366 \mathrm{pc} \\
-366 \mathrm{pc} \\
0 \mathrm{pc} \text {, at } 7 \mathrm{kpc}(3) \\
0 \mathrm{pc} \\
0 \mathrm{pc} \\
0 \mathrm{pc} \\
122 \mathrm{pc} \\
427 \mathrm{pc} \\
488 \mathrm{pc} \\
488 \mathrm{pc} \\
488 \mathrm{pc}\end{array}$ & $\begin{array}{l}\text { Downes et al (1980 - fig.4); see Table } 4 \\
\text { Chen et al (1996 - fig.1) } \\
\text { Bronfman et al (2000b - table } 2) ; \text { see Table } 3 \\
\text { Taylor \& Cordes (1993 - fig.4) } \\
\text { Englmaier \& Gerhard (1999 - table 1) } \\
\text { Beuermann et al (1985 - fig.1) } \\
\text { Bloemen et al (1990 - fig.5) } \\
\text { Caswell et al (2011 - sect. 4.6.2); see Table } 5 \\
\text { Drimmell (2000 - fig. 1) } \\
\text { Hayakawa et al (1981 - fig.2a) } \\
\text { Beuther et al (2012 - fig.3) }\end{array}$ \\
\hline $\begin{array}{l}\text { Start } \\
\text { of Per- } \\
\text { seus }\end{array}$ & $\begin{array}{l}{ }^{12} \mathrm{CO} \text { at } 8 \\
\text { FIR [CII] \& [NII] } \\
\text { Dust } 870 \mu \mathrm{m} \\
\text { Methanol masers } \\
\text { Sync. } 408 \mathrm{MHz} \\
\text { Dust } 2.4 \mu \mathrm{m} \\
\text { Dust } 60 \mu \mathrm{m}\end{array}$ & $\begin{array}{l}337^{\circ} \\
338^{\circ} \\
338^{\circ} \\
338^{\circ} \\
339^{\circ} \\
339^{\circ} \\
340^{\circ}\end{array}$ & $\begin{array}{l}00 \\
10 \\
10 \\
10 \\
20 \\
20 \\
30\end{array}$ & $\begin{array}{l}0 \mathrm{pc} \text {, at } 8 \mathrm{kpc}^{(3)} \\
140 \mathrm{pc} \\
140 \mathrm{pc} \\
140 \mathrm{pc} \\
279 \mathrm{pc} \\
279 \mathrm{pc} \\
419 \mathrm{pc}\end{array}$ & $\begin{array}{l}\text { Bronfman et al ( } 2000 \text { b - table } 2) \text {; see Table } 3 \\
\text { Steiman-Cameron et al ( } 2010 \text { - sect. } 2.1) \\
\text { Beuther et al ( } 2012 \text { - fig.3) } \\
\text { Green et al ( } 2011 \text { - sect. } 3.3 .1) \text {; see Table } 5 \\
\text { Beuermann et al (1985 - fig.1) } \\
\text { Hayakawa et al (1981 - fig.2a) } \\
\text { Bloemen et al (1990 - fig.5) }\end{array}$ \\
\hline $\begin{array}{l}\text { Near } \\
3-k p c \\
\text { Arm }\end{array}$ & $\begin{array}{l}{ }^{12} \mathrm{CO} \text { at } 8 \\
\text { HII complex } \\
\text { Warm }{ }^{12} \mathrm{CO} \text { cores }\end{array}$ & $\begin{array}{l}026^{\circ} \\
025^{\circ} \\
024^{\circ}\end{array}$ & $\begin{array}{l}00 \\
10 \\
20\end{array}$ & $\begin{array}{l}0 \mathrm{pc} \text {, at } 6 \mathrm{kpc}^{(3)} \\
105 \mathrm{pc} \\
209 \mathrm{pc}\end{array}$ & $\begin{array}{l}\text { Cohen et al (1980 - fig.3); see Table } 3 \\
\text { Russeil (2003 - table 6); see Table } 4 \\
\text { Solomon et al (1985 - fig 1b); Bania (1980 - fig.7) }\end{array}$ \\
\hline
\end{tabular}




\begin{tabular}{|c|c|c|c|c|c|}
\hline \multirow[t]{14}{*}{ Scutum } & ${ }^{12} \mathrm{CO}$ at 8 & $033^{\circ}$ & $0^{\circ}$ & $0 \mathrm{pc}$, at $5 \mathrm{kpc}^{(3)}$ & Sanders et al (1985 - fig. 5b); see Table 3 \\
\hline & HII complex & $032^{\circ}$ & 10 & $87 \mathrm{pc}$ & Russeil (2003 - Table 6); see Table 4 \\
\hline & ${ }^{26} \mathrm{Al}$ & $032^{\circ}$ & 10 & $87 \mathrm{pc}$ & Chen et al (1996 - fig.1) \\
\hline & ${ }^{13} \mathrm{CO}$ & $032^{\circ}$ & 10 & $87 \mathrm{pc}$ & Stark \& Lee $(2006-$ fig. $1, v=+95 \mathrm{~km} / \mathrm{s})$ \\
\hline & Thermal electron & $032^{\circ}$ & 10 & $87 \mathrm{pc}$ & Taylor \& Cordes (1993 - fig.4) \\
\hline & Sync. 408 MHz & $032^{\circ}$ & 10 & $87 \mathrm{pc}$ & Beuermann et al (1985 - fig.1) \\
\hline & Dust $870 \mu \mathrm{m}$ & 0310 & $2^{\circ}$ & $174 \mathrm{pc}$ & Beuther et al (2012 - fig.3) \\
\hline & Dust $240 \mu \mathrm{m}$ & 0310 & $2^{\circ}$ & $174 \mathrm{pc}$ & Drimmell $(2000$ - fig. 1) \\
\hline & Warm ${ }^{12} \mathrm{CO}$ cores & $030^{\circ}$ & 30 & 262 pc & Solomon et al (1985 - fig. 1b) \\
\hline & $\mathrm{FIR}[\mathrm{CII}] \&[\mathrm{NII}]$ & $030^{\circ}$ & 30 & $262 \mathrm{pc}$ & Steiman-Cameron et al $(2010-\operatorname{sect} 2.1)$ \\
\hline & $\mathrm{HI}$ atom & $029^{\circ}$ & $4^{\circ}$ & $349 \mathrm{pc}$ & Englmaier \& Gerhard (1999 - table 1) \\
\hline & Dust $2.4 \mu \mathrm{m}$ & $029^{\circ}$ & $4^{\circ}$ & 349 pc & Hayakawa et al (1981 - fig. 2a) \\
\hline & Methanol masers & $028^{\circ}$ & $5^{0}$ & $435 \mathrm{pc}$ & Green et al (2011 - sect. 3.3.1); see Table 5 \\
\hline & Dust $60 \mu \mathrm{m}$ & $026^{\circ}$ & 70 & 609 pc & Bloemen et al (1990 - fig.5) \\
\hline \multirow{12}{*}{$\begin{array}{l}\text { Sagit- } \\
\text { tarius }\end{array}$} & ${ }^{12} \mathrm{CO}$ at 8 & 0510 & 00 & $0 \mathrm{pc}$, at $4 \mathrm{kpc}^{(3)}$ & Cohen et al (1980 - fig.3); see Table 3 \\
\hline & HII complex & 0510 & 00 & $0 \mathrm{pc}$ & Russeil et al (2007 - fig.4); see Table 4 \\
\hline & ${ }^{13} \mathrm{CO}$ & $051^{\circ}$ & $0^{\circ}$ & $0 \mathrm{pc}$ & Stark \& Lee $(2006-$ fig. $1, v=+60 \mathrm{~km} / \mathrm{s})$ \\
\hline & $\mathrm{HI}$ atom & $050^{\circ}$ & 10 & $70 \mathrm{pc}$ & Englmaier \& Gerhard (1999 - table 1) \\
\hline & Dust $240 \mu \mathrm{m}$ & $050^{\circ}$ & 10 & $70 \mathrm{pc}$ & Drimmell $(2000$ - fig. 1) \\
\hline & Methanol masers & $050^{\circ}$ & 10 & $70 \mathrm{pc}$ & Reid et al (2014 - fig.1); see Table 5 \\
\hline & $\mathrm{FIR}[\mathrm{CII}] \&[\mathrm{NII}]$ & $050^{\circ}$ & 10 & $70 \mathrm{pc}$ & Steiman-Cameron et al (2010 - sect. 2.1) \\
\hline & Warm ${ }^{12} \mathrm{CO}$ cores & $049^{\circ}$ & $2^{\circ}$ & $140 \mathrm{pc}$ & Solomon et al (1985 - fig. 1b) \\
\hline & 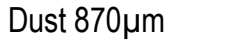 & $049^{\circ}$ & $2^{\circ}$ & $140 \mathrm{pc}$ & Beuther et al (2012 - fig.3) \\
\hline & Thermal electron & $049^{\circ}$ & $2^{\circ}$ & $140 \mathrm{pc}$ & Taylor \& Cordes (1993 - fig.4) \\
\hline & Sync. 408 MHz & $048^{\circ}$ & $3^{0}$ & $209 \mathrm{pc}$ & Beuermann et al (1985 - fig. 1) \\
\hline & ${ }^{26} \mathrm{Al}$ & $046^{\circ}$ & $5^{\circ}$ & $449 \mathrm{pc}$ & Chen et al (1996 - fig.1) \\
\hline
\end{tabular}

Notes:

(1): Galactic longitude of arm tangent, as observed by each tracer (not theoretically computed).

(2): Angular distance from arm center, being positive towards arm's inner edge (towards Galactic Center), and negative in other direction (galactic anti-center).

(3): Linear separation from the arm center $\left({ }^{12} \mathrm{CO}\right)$, after converting the angular separation at the arm distance from the sun (taken from Figure 1 here); we assume $8.0 \mathrm{kpc}$ for the distance of the Sun to the Galactic Center.

(4): When there are more than 2 published reports for a given arm tracer at a given spiral arm, then a separate table is provided in the Appendix. 
Table 2 - The linear separation $(S)$ from ${ }^{12} \mathrm{CO}$ of each arm tracer, in each spiral arm in the Milky Way ${ }^{(1)}$

\begin{tabular}{|c|c|c|c|c|c|c|c|c|c|}
\hline $\begin{array}{l}\text { Chemical } \\
\text { Tracer }\end{array}$ & $\begin{array}{l}\text { S in } \\
\text { Carina } \\
\text { arm }\end{array}$ & $\begin{array}{l}\text { S in } \\
\text { Crux } \\
\text { arm }\end{array}$ & $\begin{array}{l}\text { S in } \\
\text { Norma } \\
\text { arm } \\
\text { pc }\end{array}$ & $\begin{array}{l}\text { S in } \\
\text { Start } \\
\text { of } \\
\text { Per- } \\
\text { Seus } \\
\text { arm } \\
\text { pc }\end{array}$ & $\begin{array}{l}\text { S in } \\
\text { Near } \\
3-k p c \\
\text { arm }\end{array}$ & $\begin{array}{l}\text { S in } \\
\text { Scutum } \\
\text { arm } \\
\text { pc }\end{array}$ & $\begin{array}{l}\text { S in } \\
\text { Sa- } \\
\text { git- } \\
\text { ta- } \\
\text { rius } \\
\text { arm } \\
\text { pc }\end{array}$ & $\begin{array}{l}\text { Mean } \\
\text { sepa- } \\
\text { ration }\end{array}$ & s.d.m. ${ }^{(2)}$ \\
\hline${ }^{12} \mathrm{CO}$ at $8^{\prime}$ & 0 & 0 & 0 & 0 & 0 & 0 & 0 & 0 & $-(3)$ \\
\hline HII complex & 174 & 0 & -366 & - & 105 & 87 & 0 & 0 & 78 \\
\hline${ }^{13} \mathrm{CO}$ & - & - & - & - & - & 87 & 0 & 44 & 44 \\
\hline Thermal electron & 87 & 0 & 0 & - & - & 87 & 140 & 63 & 27 \\
\hline${ }^{26} \mathrm{Al}$ & - & 105 & -366 & - & - & 87 & 449 & 69 & 167 \\
\hline $\mathrm{HI}$ atom & - & 105 & 0 & - & - & 349 & 70 & 131 & 76 \\
\hline Synch. 408 MHz & - & 105 & 0 & 279 & - & 87 & 209 & 136 & 93 \\
\hline $\mathrm{FIR}[\mathrm{CII}] \&[\mathrm{NII}]$ & 435 & 0 & - & 140 & - & 262 & 70 & 181 & 77 \\
\hline Warm ${ }^{12} \mathrm{CO}$ cores & - & - & - & - & 209 & 262 & 140 & 204 & 35 \\
\hline Cold dust $240 \mu \mathrm{m}$ & 174 & 209 & 488 & - & - & 174 & 70 & 223 & $70(4)$ \\
\hline Cold dust $870 \mu \mathrm{m}$ & - & 209 & 488 & 140 & - & 174 & 140 & 230 & $46(4)$ \\
\hline Methanol masers & - & - & 427 & 140 & - & 435 & 70 & 268 & 95 \\
\hline Hot dust $60 \mu m$ & 262 & 209 & 122 & 419 & - & 609 & - & 324 & $86(5)$ \\
\hline Hot dust $2.4 \mu \mathrm{m}$ & - & - & 488 & 279 & - & 349 & - & 372 & $61(5)$ \\
\hline \multirow{2}{*}{\multicolumn{10}{|c|}{$\begin{array}{l}\text { Notes: } \\
\text { (1): All data from Table } 1 \text { here. }\end{array}$}} \\
\hline & & & & & & & & & \\
\hline \multicolumn{10}{|c|}{ (3): There is a mean internal scatter of $42 \mathrm{pc}$, from the $\mathrm{CO}$ data in the Appendix (Table 3). } \\
\hline \multicolumn{10}{|c|}{ (4): Stats made on both cold dust tracers give a mean separation of 227 pc, with s.d.m. of 46 pc. } \\
\hline
\end{tabular}




\section{Arm tangent offset, for each tracer}

Different arm tracers appear at slightly different galactic longitudes. To transfer angular to linear offsets, we used the canonical 4-arm logarithmic spiral model, with a Sun-Galactic Centre distance of $8.0 \mathrm{kpc}$, which is a mean of many recent measurements of this distance. The observed angular offset and computed linear separation of each individual arm tracer from the mid-arm are given, using the galactic longitude of the arm tangent for tracer $\mathrm{X}$ versus the galactic longitude of ${ }^{12} \mathrm{CO}$ arm tangent at low angular resolution.

Angle-wise, it can readily be seen in Table 1 that the hot dust arm tangents (and the methanol masers) are always inward, closer to the direction of the Galactic Center. Given enough arm tracers, and summing and averaging over all four spiral arms, one could determine if arm tracers have separate and parallel lanes in the Milky Way.

In Table 2 we pull together the statistics over several spiral arms in the Milky Way, indicating the mean separation for each arm tracer, from the mid-arm. A net separation can be found for some of the arm tracers, notably for ${ }^{12} \mathrm{CO}$ (large beam), thermal electrons (galactic free electrons, from pulsar rotation measure and dispersion measure), relativistic

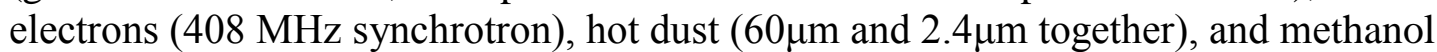
masers $(6.7 \mathrm{GHz})$. The ${ }^{26} \mathrm{Al}$ and HII complex tracers have large error bars, with a mean consistent with zero offset.

Here we make a short analysis of published stellar counts in the near infrared. The Glimpse program from the Spitzer Space Telescope has generated claims about alternating major and minor spiral arms (broad peaks in Fig.1 in Benjamin 2009; Fig. 14 and Fig.15 in Churchwell et al 2009), proposing the disappearance of the Sagittarius arm in stellar counts at $\lambda 4.5 \mu \mathrm{m}$; some authors have questioned their analysis. Steiman-Cameron (2010) and Steiman-Cameron et al (2010, Sect. 4.1) pointed out that the Sagittarius arm is observed in every major tracer, and that there is towards that galactic longitude an unusual high dust absorption, enhanced obscuration, and increased extinction (around the W51 complex). Not properly accounting for this extra dust could cause a decrease in star counts and the arm's disappearance. Also, the arm width of their major arm (Fig. 15 in Churchwell et al, 2009) is about 2.5 larger than that of their minor arm; this was questioned later. Vallée (2014a - Table 4) pointed out that the width of the Sagittarius arm is equal or nearly equal to that of the other arms, when using at least 4 different arm tracers.

\section{Galactic arm cross-cut}

\subsection{Observational results}

A crosscut of a spiral arm (Figure 2) shows the approximate position of a typical arm tracer with respect to the mid-arm, for the Milky Way. Many arms were used for the cross-cut. The direction to the Galactic Center is to the right. It is interesting that in Fig. 2 the hot dust and methanol masers are always to the right of the ${ }^{12} \mathrm{CO}$ arm tangents, while the relativistic electron and free-free electron are always in between the mid-arm and the dust lane. There is no observed spiral arm tracer between the middle of the arm and the arm's outer edge, facing the outer Galaxy, except for stars (see Table 2). 


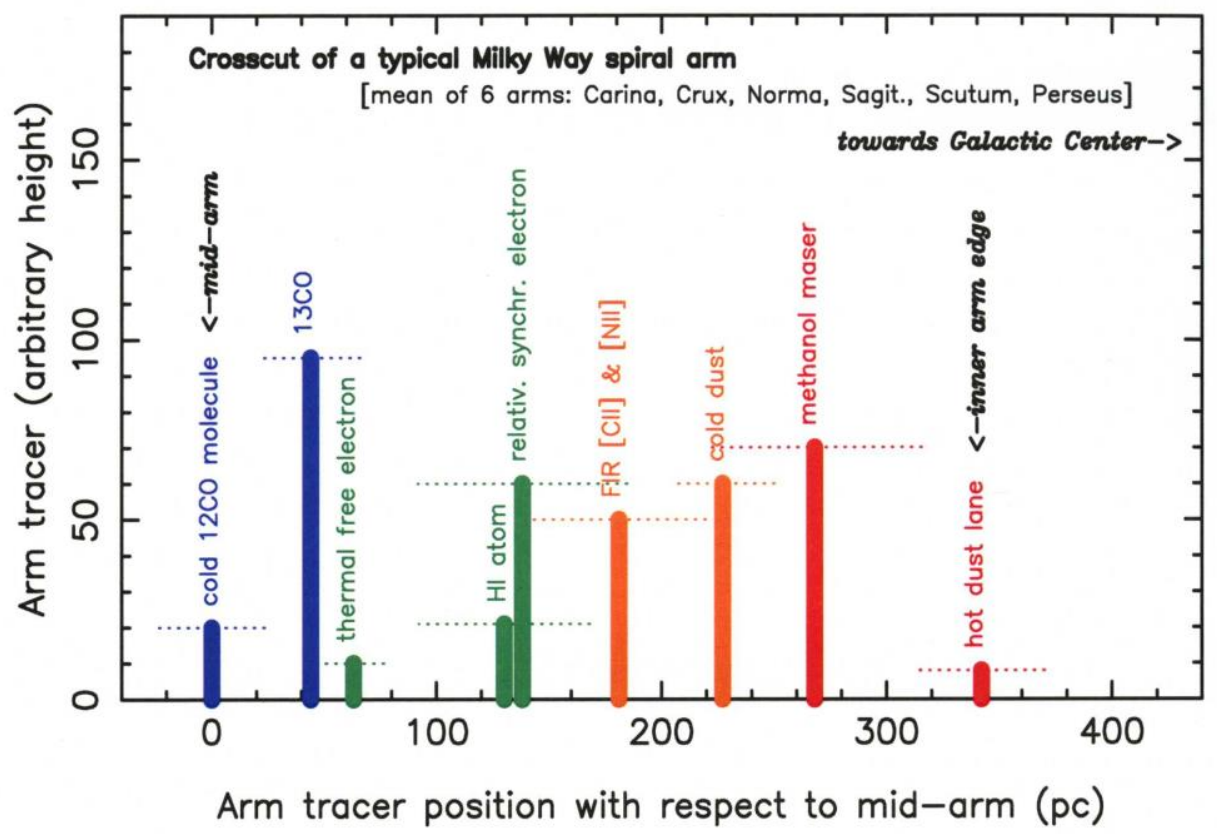

Figure 2. Average crosscut of various spiral arm in the Milky Way, showing where each different arm tracer appears. The sketch indicates the mean position of each spiral $\mathrm{arm}$, as an offset from the mid-arm $\left({ }^{12} \mathrm{CO}\right.$ tangent, at $0 \mathrm{pc}$ ) to the inner arm edge (hot dust tangent, near $340 \mathrm{pc}$ ). Vertical bars are arbitrary. The standard deviation of the mean (s.d.m.) is noted as an horizontal dotted line, for each arm tracer. Here the relativistic synchrotron is at $408 \mathrm{MHz}$. All data are from Table 2. For greater precision, the hot dust at 342 pc is an average over two wavelengths $(2.4 \mu$ and $60 \mu)$; the cold dust at $227 \mathrm{pc}$ is an average over two wavelengths $(240 \mu$ and $870 \mu)$. For greater clarity, some tracers were omitted: the average $\mathrm{HII}$ complex at $0 \mathrm{pc}$, the ${ }^{26} \mathrm{Al}$ at $69 \mathrm{pc}$, and the warm $\mathrm{CO}$ cores at $204 \mathrm{pc}$. 
The observed half width of a typical arm (near $340 \mathrm{pc}$ from mid-arm to the inner edge) can be doubled in order to get the full arm width, giving about $680 \mathrm{pc}$. The standard deviation of the mean (s.d.m.) is given for each arm tracer with the horizontal dotted lines. The mean sdm observed for the arm tracers shown (including ${ }^{12} \mathrm{CO}$ ) is near 80 pc (from Table 2), about only $12 \%$ of the full arm width.

The latest galactographic view of the Milky Way (Figure 3) shows the spiral arm lanes, using the data from Table 2 and Figure 2. On its way in a roughly circular orbit around the Galactic Center, the Sun would eventually reach the Perseus arm's hot dust lane first, and then later the next lanes (electrons; ...; CO) before exiting on the other arm's side.

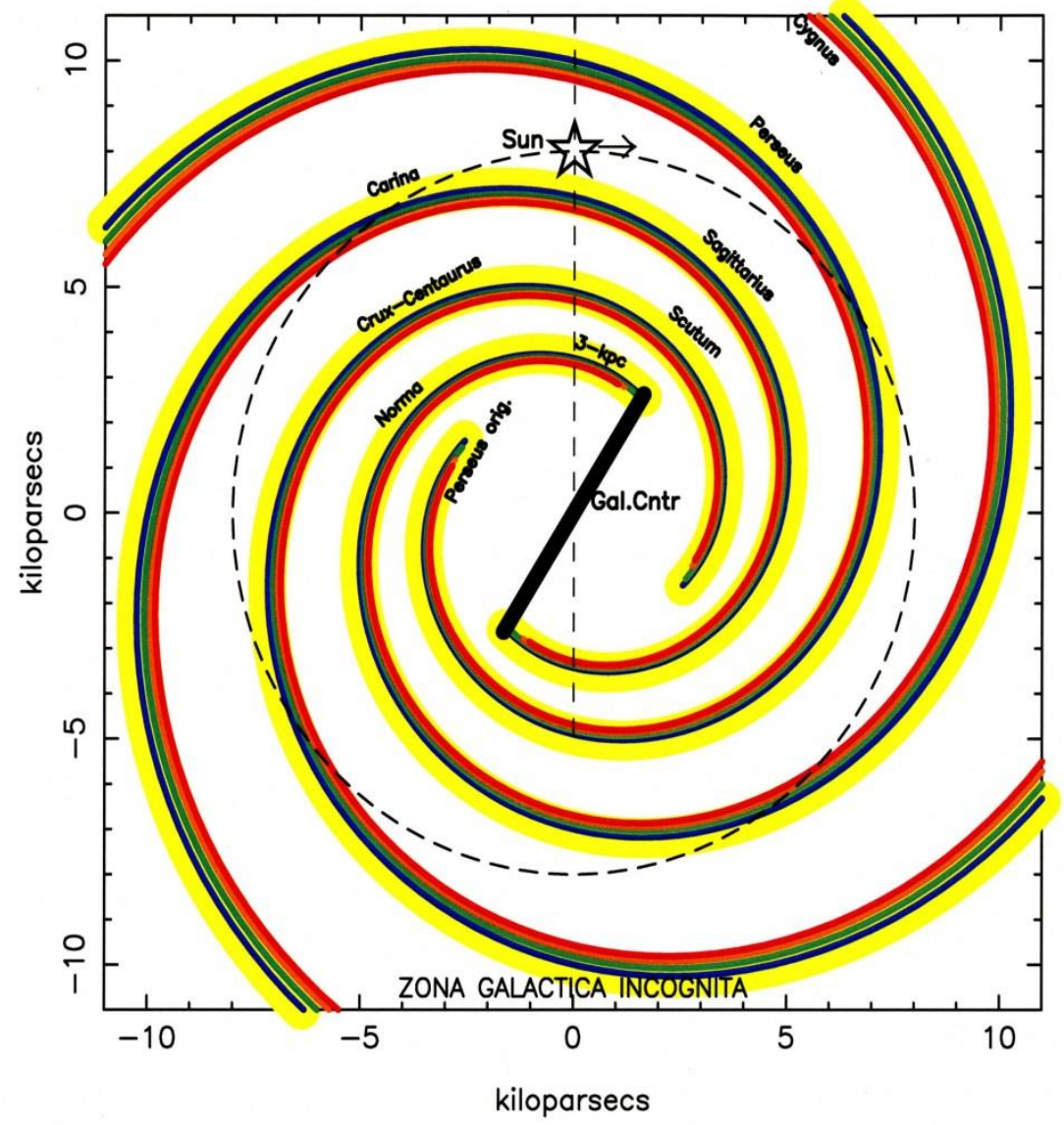

Figure 3. New galactography. Besides stars all over spiral arms, spiral arms harbor different components (tracers) at slightly at different (parallel) locations. Stars (yellow) are all over the arms. The ${ }^{12} \mathrm{CO}\left(\right.$ and $\left.\mathrm{H}_{2}\right)$ molecules peak near the middle of the arm (blue), while the hot dust (with masers and newborn stars) peak near the inner arm edge (red). Other intermediate tracers peak in between: thermal and relativistic electrons and HI atoms (green), and 
cold dust with FIR cooling lines (orange). Same color coding used as in Figure 2. Updated from Vallée (2014a).

\subsection{Comparisons with predictions from theories.}

Here we make an attempt at some quick interpretation in terms of current spiral arm theories, basically trying to relate our results with their predictions. In this brief assessment of theories of spiral arms, we compare a few observed arm parameters: linear separation of different arm tracers from ${ }^{12} \mathrm{CO}$ (table 2), no arm tracer to the left of ${ }^{12} \mathrm{CO}$ (figure 2), the number of arms (4), average pitch angle $\left(12^{\circ}\right)$, arm shape (logarithmic) and arm spacing (equal), to first order - see Vallée (2014a ).

The quasi-stationary density-wave theory can produce several arms. (i) The density wave theory predicts an orbital trajectory of the gas and stars going through spiral arms, and a linear separation between arm tracers as occasioned by a shocked gas and dust entering the arm from the inner edge, then some of it coalescing to form stars there (dust lane), progressing through the arm and exiting on the arm's outer edge (Roberts, 1975 - fig.3); this is similar to our orbit in Figure 1 here. (ii) In the density wave, the width of the dust lane (shocked lane) was predicted at 50 pc (e.g., Dobbs \& Baba 2014, their sect. 3.5); this width is similar to our observed dust lane in Figure 2 here (with an error bar of $70 \mathrm{pc}$ ). (iii) In the density wave, the gas density maximum (at mid-arm) is separated from the shock lane by about 12 Myrs (Roberts, 1975 - Fig.2), or 306 pc for a gas velocity difference of $25 \mathrm{~km} / \mathrm{s}$ (Dobbs \& Baba 2014 - fig. 17b); this separation is similar to our observed arm's halfwidth of 360 pc (Table 2 here). Roberts (1975 - Fig.4) used a pitch angle near $10^{\circ}$, similar to that of the Milky Way. In the density-wave with 4 arms, the gas density maximum (potential minimum, broad $\mathrm{CO}$ at mid-arm) is also separated from the shock lane (hot dust) in the model of Gittins and Clarke (2004 - fig.16), and their 'standard model' has a low pitch angle of $5^{\circ}$ (not the $12^{\circ}$ found in the Milky Way). (iv) The density wave theory proposed that the peak of all tracers are between the inner edge of an arm and the mid-arm (as seen here in Table 2), excepting the stars distributed all over the arm. (v) The density wave predicts that the peak of the HII complex is located near the mid-arm (Roberts 1975- fig.4, Gittins \& Clarke 2004 - fig.16 far from the Galactic Center), as seen in Table 2 here.

There is a plethora of theories to generate spiral arms, in addition to the density-wave theory. (vi) The tidal theory involving a recent passage of the Sagittarius galaxy could induce two strong arms (Dobbs \& Baba (2014, sect. 5.3), not four as found in the Milky Way. Of course, each of two Magellanic Clouds could perhaps produce its own set of twin arms. (vii) The bar-driven theory could induce two strong arms (Dobbs \& Baba 2014 - sect.2.3), not four as found in the Milky Way. Of course, each of two straddling bars could perhaps produce its own set of twin arms. (viii) The stochastic, self-propagating star formation theory could produce a very irregular, flocculent arm pattern (Dobbs \& Baba - sect. 2.5), not the quasi-regular pattern seen in the Milky Way. (ix) The swing-amplification theory could produce numerous flocculent arms irregularly located (Dobbs \& Baba - sect. 5.2), not quasi-regularly spaced as for the Milky Way. (x) The transient-recurrent dynamic spiral theory (Dobbs \& Baba 2014 - Sect. 2.2) has arms breaking and reconnecting, and high pitch angle between $20^{\circ}$ and $40^{\circ}$ (Dobbs \& Baba 2014 - fig.10), unlike the low $12^{\circ}$ as found generally in the Milky Way. (xi)The basic dynamo theory 
does not predict any spiral arm as defined here (with dust and stars), contrary to observations, although it can produce magnetic arms - for a discussion, see Vallée (2011 - Sect. 9.7). In the presence of gaseous spiral arms, non-linear galactic dynamos predict that the interarm magnetic pitch angle will be compressed to roughly align with the gaseous arm pitch angle (Chamandy et al 2014 - sect. 7). (xii) The basic MHD theory, subjected to a spiral potential, predicts different pitch angle of the magnetic field (along the gas velocity) and that of the spiral arm (Dobbs \& Price - fig.13); this is not at odd with some magnetic field observations - for a discussion, see Vallée (2011 - Sect.9.7.3).

In summary, the arm observations so far may be best compared to the density waves (iv), but many other proposed theories could be partially contributing to the creation or maintenance over time of spiral arms or spurs (vi-xii). How much from each is unknown.

\section{Conclusion}

Our place in the Milky Way galaxy (Figure1), inside the flat galactic disk, makes it difficult to see the different spiral arms, being one behind another. Strong dust absorption in the disk does reduce our view at optical wavelengths. Still, radio, infrared and optical telescopes, equipped with spectrometers and other instruments, have allowed a picture to emerge with 4 long spiral arms where most of the gas and stars are positioned and their radial velocity measured. We presented here an updated and revised Catalogue of the mean data on the different spiral arm tracers (Table 1) and their accumulations (Table 3, Table 4, Table 5), covering 105 tracer entries.

We thus confirm here a separation between arm tracers (Table 2, Fig. 2), as claimed earlier by Vallée (2014a) with fewer tracers. In the Milky Way, each spiral arm tracer can occupy a parallel lane inside an arm - there is roughly $340 \mathrm{pc}$ separation between the mid-arm $\left({ }^{12} \mathrm{CO}\right.$ tracer lane) and the inner arm edge toward the Galactic Center (hot dust lane). This provides us a fascinating view of the inside of a typical spiral arm in the Milky Way galaxy, despite the Sun's awkward position inside the flat galactic disk (Figure 3). Our interpretation in terms of the many proposed spiral arm theories is still sketchy (section 4.2), while currently favoring the hunt for the density waves.

\section{Acknowledgements.}

The figure production made use of the PGPLOT software at the NRC-nsip in Victoria. I thank a referee for insightful and helpful comments, which helped to correct and improve the clarity of the text.

\section{Appendix A.}

Here are some of the published individual tracer values, when there is more than one for each tracer in each spiral arm.

Table 3 lists numerous individual CO entries (not earlier than 1980).

Table 4 provides individual HII complex entries (not earlier than 1980).

Table 5 gives individual maser entries (not earlier than 1980). 
Table 3 - Published CO J=1-0 results at low angular resolution (since 1980)

$\begin{array}{llll}\text { Arm name } & \begin{array}{l}\text { Tangent } \\ \text { Longitude }\end{array} & \begin{array}{l}\text { Telescope HPBW } \\ \text { and survey name }\end{array} & \text { Reference }\end{array}$

\begin{tabular}{|c|c|c|c|c|}
\hline \multirow[t]{6}{*}{ Carina } & $280^{\circ}$ & $8.8^{\prime}$ & Columbia & Alvarez et al (1990 - table 4) \\
\hline & $280^{\circ}$ & $8.8^{\prime}$ & Columbia & Grabelsky et al (1987 - sect. 3.1.2) \\
\hline & $281^{\circ}$ & $8.8^{\prime}$ & Columbia & Grabelsky et al (1988 - fig.4) \\
\hline & $282^{\circ}$ & $8.8^{\prime}$ & Columbia & Bronfman et al (2000b - table 2) \\
\hline & $283^{\circ}$ & $8.8^{\prime}$ & Columbia & Bronfman et al (2000a - Section 3.4) \\
\hline & $281.2 \pm 1.3$ & \multicolumn{2}{|c|}{ mean and r.m.s. } & s.d.m of $0.6^{\circ}$, worth $51 \mathrm{pc}$ at $5 \mathrm{kpc}$ \\
\hline Crux- & $308^{\circ}$ & $8.8^{\prime}$ & Columbia & Bronfman et al (2000a - sect. 3.4) \\
\hline Cen- & $308^{\circ}$ & $8.8^{\prime}$ & Columbia & Bronfman (2008 - sect. 4) \\
\hline \multirow[t]{8}{*}{ Taurus } & $309^{\circ}$ & $8.4^{\prime}$ & CfA & Dame \& Thaddeus (2011 - fig.4) \\
\hline & $309^{\circ}$ & $8.8^{\prime}$ & Columbia & Bronfman et al (2000b - table 2) \\
\hline & $310^{\circ}$ & $8.8^{\prime}$ & Columbia & Alvarez et al (1990 - table 4) \\
\hline & $310^{\circ}$ & $8.8^{\prime}$ & Columbia & Bronfman et al (1988 - fig. 7) \\
\hline & $310^{\circ}$ & $8.8^{\prime}$ & Columbia & Bronfman et al (1989- sect. 4) \\
\hline & $310^{\circ}$ & $8.8^{\prime}$ & Columbia & García et al (2014 - table 3) \\
\hline & $310^{\circ}$ & $8.8^{\prime}$ & Columbia & Grabelsky et al (1987 - sect. 3.1.2) \\
\hline & $309.3 \pm 0.9$ & \multicolumn{2}{|c|}{ mean and r.m.s. } & s.d.m of $0.3^{\circ}$, worth $34 \mathrm{pc}$ at $6 \mathrm{kpc}$ \\
\hline \multirow[t]{10}{*}{ Norma } & $328^{\circ}$ & $8.8^{\prime}$ & Columbia & Alvarez et al (1990 - table 4) \\
\hline & $328^{\circ}$ & $8.8^{\prime}$ & Columbia & Bronfman et al (1988 - fig. 7) \\
\hline & $328^{\circ}$ & $8.8^{\prime}$ & Columbia & Bronfman et al (1989- sect. 4) \\
\hline & $328^{\circ}$ & $8.8^{\prime}$ & Columbia & Bronfman (1992 - fig.6) \\
\hline & $328^{\circ}$ & $8.8^{\prime}$ & Columbia & Bronfman et al (2000a - sect. 3.4) \\
\hline & $328^{\circ}$ & $8.8^{\prime}$ & Columbia & Bronfman et al (2000b - table 2$)$ \\
\hline & $328^{\circ}$ & $8.8^{\prime}$ & Columbia & Bronfman (2008 - sect. 4) \\
\hline & $330^{\circ}$ & $8.8^{\prime}$ & Columbia & García et al (2014 - table 3) \\
\hline & $330^{\circ}$ & $8.8^{\prime}$ & Columbia & Grabelsky et al (1987 - sect. 3.1.2) \\
\hline & $328.4 \pm 0.9$ & \multicolumn{2}{|c|}{ mean and r.m.s. } & s.d.m of $0.4^{\circ}$, worth $37 \mathrm{pc}$ at $7 \mathrm{kpc}$ \\
\hline Start & $336^{\circ}$ & $8.8^{\prime}$ & Columbia & Bronfman et al (1989 - sect. 4) \\
\hline of Per- & $336^{\circ}$ & $8.8^{\prime}$ & Columbia & Bronfman (2008 - sect. 4) \\
\hline \multirow[t]{5}{*}{ seus } & $337^{\circ}$ & $8.8^{\prime}$ & Columbia & Alvarez et al (1990 - table 4) \\
\hline & $337^{\circ}$ & $8.8^{\prime}$ & Columbia & Bronfman et al (2000a - sect. 3.4) \\
\hline & $337^{\circ}$ & $8.8^{\prime}$ & Columbia & Bronfman et al (2000b - table 2) \\
\hline & $337^{\circ}$ & $8.8^{\prime}$ & Columbia & Dame \& Thaddeus (2008 - sect. 1 ) \\
\hline & $338^{\circ}$ & $8.8^{\prime}$ & Columbia & García et al (2014 - table 3) \\
\hline
\end{tabular}




$336.9 \pm 0.7 \quad$ mean and r.m.s. s.d.m of $0.3^{\circ}$, worth $37 \mathrm{pc}$ at $8 \mathrm{kpc}$

\begin{tabular}{|c|c|c|c|c|}
\hline \multirow[t]{2}{*}{$\begin{array}{l}\text { Near 3-kpc } \\
\text { arm }\end{array}$} & $\begin{array}{l}026^{\circ} \\
026^{\circ}\end{array}$ & $\begin{array}{l}7.5^{\prime} \\
7.5^{\prime}\end{array}$ & $\begin{array}{l}\text { Columbia } \\
\text { Columbia }\end{array}$ & $\begin{array}{l}\text { Cohen et al (1980 - fig.3) } \\
\text { Dame et al (1986 - fig.9) }\end{array}$ \\
\hline & $026^{\circ}$ & & mean & \\
\hline \multirow[t]{5}{*}{ Scutum } & $031^{\circ}$ & $8.4^{\prime}$ & CfA & Dame \& Thaddeus (2011 - fig.4) \\
\hline & $033^{\circ}$ & $1.1^{\prime}$ & NRAO & Sanders et al (1985 - fig.5b) \\
\hline & $034^{\circ}$ & $7.5^{\prime}$ & Columbia & Cohen et al (1980 - fig.3) \\
\hline & $035^{\circ}$ & $1.0^{\prime}$ & Texas & Chiar et al (1994 - Sect. 3) \\
\hline & $033.2 \pm 1.7$ & \multicolumn{2}{|c|}{ mean and r.m.s. } & s.d.m of $0.8^{\circ}$, worth $70 \mathrm{pc}$ at $5 \mathrm{kpc}$ \\
\hline \multirow[t]{2}{*}{ Sagittarius } & $051^{\circ}$ & 7.5' & Columbia & Cohen et al (1980 - fig.3) \\
\hline & $051^{\circ}$ & $7.5^{\prime}$ & Columbia & Dame et al (1986 - fig.9) \\
\hline
\end{tabular}


$\underline{\text { Table 4-Published HIl complex results }}$

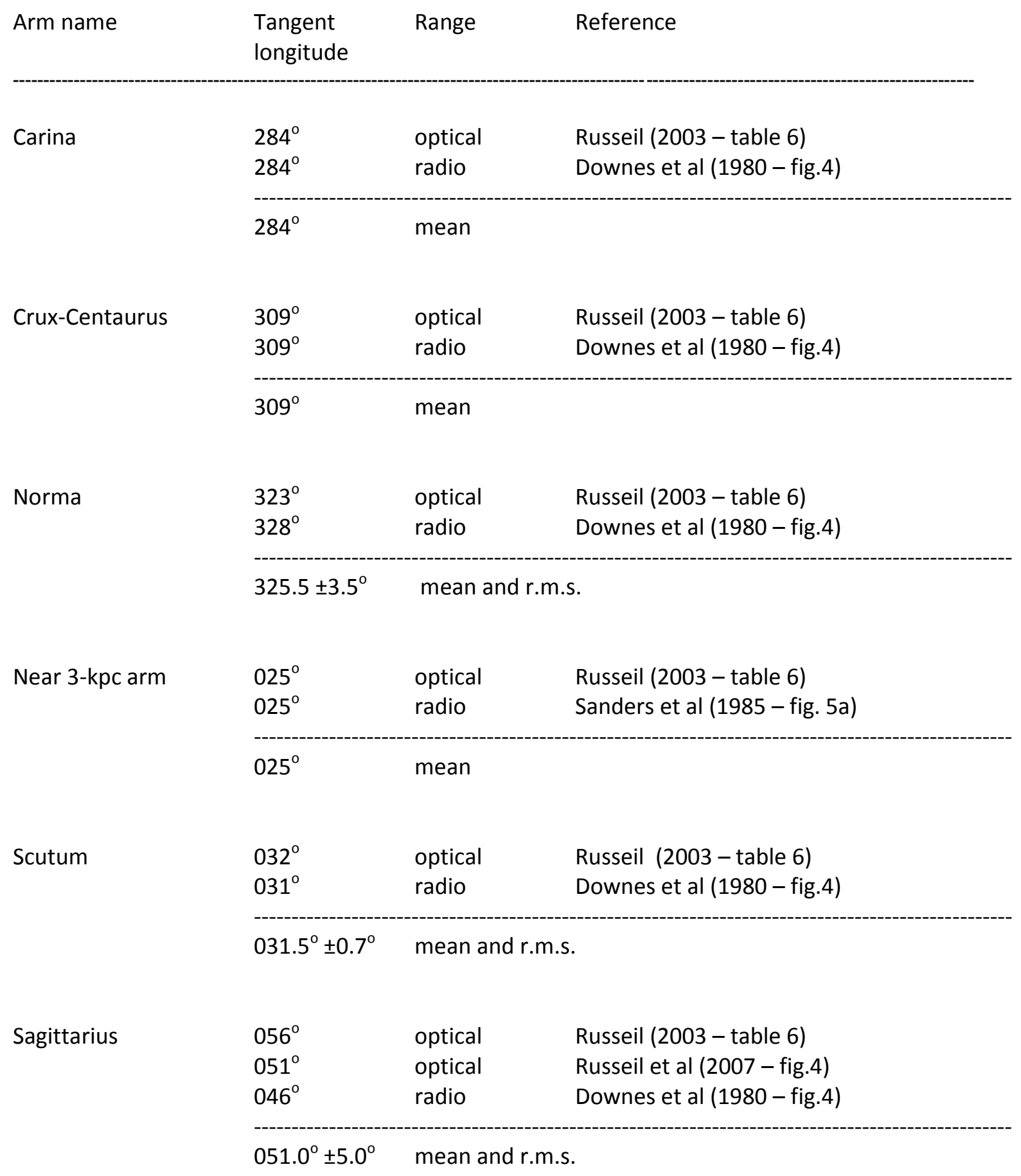


$\underline{\text { Table 5-Published maser results }}$

\begin{tabular}{|c|c|c|c|}
\hline Arm name & $\begin{array}{l}\text { Tangent } \\
\text { longitude }\end{array}$ & Range & Reference \\
\hline \multirow[t]{2}{*}{ Norma } & $331.5^{\circ}$ & methanol & Caswell et al (2011 - Sect. 4.6.2) \\
\hline & $331.5^{\circ}$ & mean & \\
\hline \multirow[t]{2}{*}{ Start of Perseus } & $\begin{array}{l}338^{\circ} \\
338^{\circ}\end{array}$ & $\begin{array}{l}\text { methanol } \\
\text { methanol }\end{array}$ & $\begin{array}{l}\text { Green et al (2011 - sect. 3.3.1) } \\
\text { Green et al (2012 - Sect. } 2)\end{array}$ \\
\hline & $338.0^{\circ}$ & mean & \\
\hline \multirow[t]{2}{*}{ Scutum } & $\begin{array}{l}026^{\circ} \\
026^{\circ} \\
030^{\circ} \\
031^{\circ}\end{array}$ & $\begin{array}{l}\text { methanol } \\
\text { methanol } \\
\text { methanol, water } \\
\text { methanol, water }\end{array}$ & $\begin{array}{l}\text { Green et al (2011 - sect. 3.3.1) } \\
\text { Green et al ( } 2012 \text { - Sect. } 2) \\
\text { Reid et al ( } 2014-\text { fig.1) } \\
\text { Sato et al ( } 2014 \text { - fig3) }\end{array}$ \\
\hline & $028.2^{\circ} \pm 2.6^{\circ}$ & mean and r.m.s. & \\
\hline Sagittarius & $\begin{array}{l}049.6^{\circ} \\
050^{\circ} \\
051^{\circ}\end{array}$ & $\begin{array}{l}\text { methanol } \\
\text { methanol, water } \\
\text { methanol, water }\end{array}$ & $\begin{array}{l}\text { Pandian \& Goldsmith (2007 - sect.4) } \\
\text { Reid et al ( } 2014 \text { - fig. } 1) \\
\text { Wu et al (2014 - sect. } 4.2)\end{array}$ \\
\hline
\end{tabular}




\section{References:}

Alvarez, H. May, J., Bronfman, L., "The rotation of the Galaxy within the solar circle",1990, Astrophys. J., v348, p495-502.

Bania, T.M., "Carbon Monoxyde in the inner Galaxy: the 3-kpc arm and otherexpanding features", 1980, ApJ, v242, p95-111.

Benjamin, R.A., "Spiral arm tangencies in the Milky Way", 2009, Proc. IAU Symp., v254, p319-322.

Beuermann, K., Kanbach, G., Berkhuijsen, E.M., "Radio structure of the Galaxy - Thick disk and thin disk at 408 MHz", 1985, A\&A, v153, p17-34.

Beuther, H., Tackenberg, J., Linz, H., Henning, Th., Schuller, F., Wyrowski, F, Schilke,P., Menten, K., Robitaille, T.P., Wlamsley, C.M., Bronfman, L., Motte, F., Nguyen-Luong, Q., Bontemps, S., "Galactic structure based on the Atlasgal $870 \mu$ m survey", 2012, ApJ, v747, a43, p1-8.

Bloemen, J.B., Deul, E.R., Thaddeus, P., "Decomposition of the FIR Milky Way observed by IRAS", 1990, A\&A, v233, p437-455.

Bronfman , L., "Molecular clouds and young massive stars in the Galactic disk", 1992, Astrophys Space Sci Lib., v180, p131-154.

Bronfman, L. "Massive star formation in the southern Milky Way", 2008, Ap Sp Sci., v313, p81-85.

Bronfman, L., Alvarez, H., Cohen, R.S., Thaddeus, P., "A deep CO survey of molecular clouds in the southern Milky Way", 1989, ApJ Suppl Ser., v71, p481-548.

Bronfman, L., Casassus, S., May, J., Nyman, L.-A., "The radial distribution of OB star formation in the Galaxy", 2000a, Astron. \& Astrophys., v358, p521-534.

Bronfman, L., Cohen, R.S., Alvarez, H., May, J., Thaddeus, P., "A CO survey of the southern Milky Way: the mean radial distribution of molecular clouds within the solar circle", 1988, ApJ, v324, p248-266.

Bronfman, L., May, J., Luna, A., "A CO survey of the southern Galaxy”, 2000b, ASP Confer. Ser., v217, p66-71.

Caswell, J.L., Fuller, G.A., Green, J.A., Avison, A., Breen, S.L., Ellingsen, S.P., Gray, M.D., Pestalozzi, M.R., Quinn, L., Thompson, M.A., Voronkon, M.A., "The $6 \mathrm{GHz}$ methanol multibeam maser catalogue - III. Galactic longitudes 330 o to $3450 ", 2011$, MNRAS, v417, p1964-1995.

Chamandy, L., Shukurov, A., Subramanian, K., Stoker, K., "Non-linear galactic dynamos : a toolbox", 2014, MNRAS, v443, p1867-1880.

Chen,W., Gehrels, N., Diehl, R., Hartmann, D., "On the spiral arm interpretation of Comptel ${ }^{26} \mathrm{Al}$ map features", 1996, A\&A Suppl., v120, p315.

Chiar, J.E., Kutner, M.L., Verter, F., Leous, J., "A comparison of CO ( $\mathrm{J}=1-0)$ and $\mathrm{CO}(\mathrm{J}=2-1)$ emission in the Milky Way molecular ring", 1994, ApJ, v431, p658-673. 
Churchwell, E., Babler, L.B.,, Meade, M.R., Whitney, B.A., Benjamin, R., Indebetouw, R., Cyganowski, C., Robitaille, T.P., Povich, M., Watson, C., Bracker, S., "The Spitzer/GLIMPSE surveys: a new view of the Milky Way", 2009, Publ. Astron. Soc. Pacific, v121, p213-230.

Cohen, R.S., Cong, H., Dame, T.M., Thaddeus, P., "Molecular clouds and galactic spiral structure", 1980, ApJ, v239, L53-L56.

Dame, T.M., Elmegreen, B.G., Cohen, R.S., Thaddeus, P., "The largest molecular cloud complexes in the first galactic quadrant", 1986, ApJ, v305, p892-908.

Dame, T.M., Thaddeus, P., "A new spiral arm of the Galaxy: the Far 3kpc arm", 2008, ApJ, v683, L143-L146.

Dame, T.M., Thaddeus, P., "A molecular spiral arm in the far outer galaxy", 2011, ApJL, v734, L24, p1-4.

Dobbs, C., Baba, J., "Dawes review 4 : spiral structures in disc galaxies", 2014, Publ Astron Soc Australia, in press astro-ph.GA arXiv:1407.5062

Dobbs, C.L., A. Burkert, "The myth of the molecular ring", 2012, Monthly Notices Royal Astronomical Society, v421, p2940-2946.

Dobbs, C.L., Price, D.J., "Magnetic fields and the dynamics of spiral galaxies", 2008, MNRAS, v383, p497-512.

Downes, D., Wilson, T.L., Bieging, J., Wink, J., " $\mathrm{H} 110 \mathrm{a}$ and $\mathrm{H}_{2} \mathrm{CO}$ survey of galactic radio sources", 1980, A\&A Suppl., v40, p379-394.

Drimmel, R., "Evidence for a 2-armed spiral in the Milky Way", 2000, A\&A, v358, L13-L16.

Englmaier,P., Gerhard,O., " Gas dynamics and large-scale morphology of the Milky Way galaxy ", 1999, MNRAS, v304, p512-534.

García, P., Bronfman, L., Nyman, L.-A., Dame, T.M., Luna, A., " Giant molecular clouds and massive star formation in the southern Milky Way", 2014, AstrophysJSuppl.Ser., v212, a2, p1-33.

Gittins, D.M., Clarke, C.J., "Constraining corotation from shocks in tightly wound spiral galaxies", 2004, MNRAS, v349, p909-921.

Grabelsky, D.A., Cohen, R.S., Bronfman, L., Thaddeus, P., May, J., " Molecular clouds in the Carina arm largescale properties of molecular gas and comparison with HI”, 1987, Astrophysical journal, v315, p122-141.

Grabelsky, D.A., R.S. Cohen, L.Bronfman, P.Thaddeus, " Molecular clouds in the Carina arm - the largest objects, associated regions of star formation, and the Carina arm in the Galaxy", 1988, Astrophysical journal, v331, p181-196.

Green, J.A., Caswell, J.L., McClure-Griffiths, N.M., Avison, A., Breen, S.L., Burton, M.G., Ellingsen, S.P., Fuller, G.A., Gray, M.D., Pestalozzi, M., Thompson, M.A., Voronkov, M.A., "Major structures of the inner Galaxy delineated by 6.7GHz methanol masers", 2011, ApJ, v733, a27, p1-17.

Green, J.A., Caswell, J.L., McClure-Griffiths, N.M., Avison, A., Breen, S.L., Burton, M.G., Ellingsen, S.P., Fuller, G.A., Gray, M.D., Pestalozzi, M., Thompson, M.A., Voronkov, M.A., "Tracing major structures of the inner Galaxy with 6.7GHz methanol masers", 2012, EPJ Web of conferences, v19, a06007, p1-3. 
Hayakawa, S., Matsumoto, T., Murakami, H., Uyama, K., Thomas, J.A., Yamagami, T., "Distribution of near infrared sources in the galactic disk", 1981, A\&A, v100, p116.

Heiles, C., Haverkorn, M., "Magnetic fields in the multiphase interstellar medium", 2012, Space Sci Rev., v166, p293305.

Martinez-Valpuesta, I., "A bar/bulge model for the Milky Way", 2013, in Highlights of Spanish Astrophysics VII, Proc. of the X Sci. Mtg, of the SEA, ed. by Guirado, J.C., Lara, L.M., Quilis, V., Gorgas, J. (Sociedad Espanola de Astronomia, Barcelona, Spain), p484-491.

Pandian, J.D., Goldsmith, P.E., "The Arecibo methanol maser galactic plane survey. II. Statistical and multiwavelength counterpart analysis", 2007, ApJ, v669, p435-445.

Reid, M.J., Menten, K.M., Brunthaler, A., Zheng, X.W., Dame, T.M., Xu, Y., Wu, Y., Zhang, B., and 8 others, "Trigonometric parallaxes of high mass star forming regions: the structure and kinematics of the Milky Way", 2014, ApJ, v783, a130, p1-14.

Roberts, W.W., "Theoretical aspects of galactic research", 1975, Vistas in Astron., v19, p91-109.

Romero-Gómez, M., Antoja, T., Figueras, F., Aguilar, L.A., Athanassoula, E., " The invariant manifolds and the Milky Way galactic bar(s)", 2013, in Highlights of Spanish Astrophysics VII, Proc. of the X Sci. Mtg, of the SEA, ed. by Guirado, J.C., Lara, L.M., Quilis, V., Gorgas, J. (Sociedad Espanola de Astronomia, Barcelona, Spain), p618-622.

Russeil, D.,"Star-forming complexes and the spiral structure of our Galaxy", 2003, A\&A, v397, p133-146.

Russeil, D., Adami, C., Georgelin, Y.M., "Revised distances of Northern HII regions", 2007, A\&A, v470, p161-171.

Sanders, D.B., Scoville, N.Z., Solomon, P.M., "Giant molecular couds in the Galaxy. II. Characteristics of discrete features", 1985, ApJ, v289, p373-387.

Sato, M., Wu, Y.W., Immer, K., Zhang, B., Sanna, A., Reid, M.J., Dame, T.M., Brunthaler, A., Menten, K.M., "Trigonometric parallaxes of star forming regions in the Scutum spiral arms", 2014, ApJ, v793, a72, p1-15.

Solomon, P.M., Sanders, D.B., Rivolo, A.R., "The Massachusetts-Stony-Brook galactic plane CO survey: disk and spiral arm molecular cloud populations", 1985, ApJ, v292, L19-L24.

Stark, A.A., Lee, Y., "Giant molecular clouds are more concentrated toward spiral arms than smaller clouds", 2006, ApJ, v641, L113-L116.

Steiman-Cameron, T.Y., "Twin masks of spiral structure? A local perspective", in Galaxies and their masks, ed. by Block, D.L., Freeman, K.C., Puerari,I., Springer, 2010, p45-58.

Steiman-Cameron,, T.Y., Wolfire, M., Hollenbach, D., "COBE and the galactic interstellar medium: geometry of the spiral arms from FIR cooling lines", 2010, ApJ, v722, p1460-1473.

Taylor, J.H., Cordes, J.M., "Pulsar distances and the galactic distribution of free electron", 1993, ApJ, v411, p674684.

Vallée, J.P., " The Milky Way's Spiral Arms Traced by Magnetic Fields, Dust, Gas, and Stars", 1995, ApJ, v454, p119-124. 
Vallée, J.P., « Metastudy of the Spiral Structure of Our Home Galaxy”, 2002, ApJ, v566, p261-266.

Vallée, J.P., «The Spiral Arms and Interarm Separation of the Milky Way: An Updated Statistical Study", 2005, AJ, v130, p569-575.

Vallée, J.P., « New Velocimetry and Revised Cartography of the Spiral Arms in the Milky Way-A Consistent Symbiosis", 2008, AJ, v135, p1301-1310.

Vallée, J.P., "Magnetic field in the Galactic Universe, as observed in supershells, galaxies, intergalactic and cosmic realms", 2011, New Astron Rev., v55, p91-154.

Vallée, J.P., "Magnetic Milky Way", 2012, Europ. Astron. Soc. Publ. Ser., v56, p81-86.

Vallée, J.P., "A synthesis of fundamental parameters of spiral arms, based on recent observations in the Milky Way", 2013, IJAA, v3, p20-28.

Vallée, J.P., "The spiral arms of the Milky Way: the relative location of each different arm tracer, within a typical spiral arm width", 2014a, Astron,J., v.148, a5, p1-9.

Vallée, J.P., "On a persistent large discrepancy in some observed parameters of the spiral arm in the Milky Way - a statistical and modelling analysis", 2014b, MNRAS, v442, p2993-2998.

Wu, Y.W., Sato, M., Reid, M.J., Moscadelli, L., Zhang, B., Xu, Y., Brunthaler, A.,Menten, K.M., Dame, T.M., Zheng, X.W., "Trigonometric parallaxes of star forming regions in the Sagittarius spiral arm", 2014, A\&A, v566, a17, p1-26. 